\title{
Composición Corporal y Somatotipo de Rugbistas Chilenos y su Relación con la Posición de Juego
}

\author{
Body Composition and Somatotype of Chilean Rugby Players \\ and their Relationship with the Game Position
}

Báez-San Martín, E. '; Jil-Beltrán, K.²; Ramírez-Campillo, R. ${ }^{3}$; Tuesta M. ${ }^{4}$; Barraza-Gómez, F. ${ }^{5}$; Opitz-Ben-Hour, A. ${ }^{6}$ \& Yáñez-Sepúlveda, R. ${ }^{7}$

\begin{abstract}
BÁEZ-SAN MARTÍN, E.; JIL-BELTRÁN, K.; RAMÍREZ-CAMPILLO, R.; TUESTA, M.; BARRAZA-GÓMEZ, F.; OPITZBEN-HOUR, A. \& YÁÑEZ-SEPÚLVEDA, R. Composición corporal y somatotipo de rugbistas chilenos y su relación con la posición de juego. Int. J. Morphol., 37(1):331-337, 2019.
\end{abstract}

RESUMEN: El objetivo del estudio fue describir las características morfoestructurales, por posición de juego, en rugbistas de la región de Valparaíso, Chile. La estatura, peso, índice de masa corporal (IMC) y otros 25 parámetros antropométricos fueron obtenidos desde 121 rugbistas competitivos (24,2 $\pm 4,9$ años), siguiendo los estándares de medición de la Sociedad Internacional para el Avance de la Kinantropometría (ISAK). La composición corporal fue descrita en 5 componentes a través del método propuesto por Kerr, estos son masa muscular, adiposa, residual, ósea y piel. El uso del somatotipo descrito por Carter y Heath, permitió describir los componentes de endomorfía, mesomorfía y ectomorfía. Para comparar las características morfoestructurales de los rugbistas por posición de juego, estos fueron separados en forwards y backs. El peso corporal, la estatura sentado y de pie, el IMC y la sumatoria de 8 pliegues fueron mayores en los forwards que los backs $(\mathrm{p}<0,0001)$. En relación a las masas corporales, el tejido adiposo y muscular absoluto fueron mayores en los forwards que en los backs ( $\mathrm{P}<0,01)$, no así el relativo. El somatotipo evidenció mayor endomorfía y mesomorfía, y menor ectomorfía, en los forwards 4,8-6,9-0,7 (1,8-1,3-0,8) que los backs 3,7-6,1-1,2 (1,8-1,1-0,8) con P<0,01. En conclusión, la posición de juego en el rugby es determinante en la definición de características físicas básicas y morfológicas. El mayor peso corporal de los forwards respecto a los backs se encuentra influenciado por la presencia de más tejido muscular y adiposo. Esto último se relacionó con un mayor componente mesomórfico y endomórfico del somatotipo, y menor del ectomórfico, en los forwards. A pesar de estas diferencias ambos grupos se encuentran en una clasificación de endo-mesomorfo.

PALABRAS CLAVE: Rugby, antropometría, somatotipo, composición corporal.

\section{INTRODUCCIÓN}

Es reconocido que la práctica deportiva modela las características físicas del sujeto de acuerdo a su especificidad con el fin de potenciar el rendimiento durante la competencia (Norton \& Olds, 1996). En este sentido, el estudio de la morfología del deportista, a través de la composición corporal y el somatotipo, ha sido utilizado como estrategia de selección deportiva, elección de la posición de juego, especificidad técnica-táctica, entre otras. En el rugby por ejemplo, la composición corporal de deportistas de alto rendimiento se caracteriza por un gran desarrollo del tejido muscular (Gabbett, 2000), ya que el éxito deportivo en esta dis- ciplina se encuentra estrechamente relacionado con la fuerza, potencia y agilidad (Gabbett, 2005). Cabe recordar que los movimientos corporales en el rugby durante una competencia se caracterizan por ser de alta intensidad con recuperaciones incompletas, desplegando una gran cantidad de energía anaeróbica (Higham et al., 2013). Además, la elevada frecuencia de contactos de alta intensidad con el contrincante ( 40 contactos por partido), ya sea en aspectos ofensivos o defensivos, también se verá beneficiada por un mayor componente de tejido muscular (Brewer \& Davis, 1995). A pesar de esto, las exigencias físicas según la posición de

\footnotetext{
${ }^{1}$ Departamento de Deportes y Recreación, Facultad de Ciencias de la Actividad Física, Universidad de Playa Ancha, Valparaíso, Chile.

${ }^{2}$ Laboratorio de Ciencias del Ejercicio, Salud y Rendimiento, Valparaíso, Chile.

${ }^{3}$ Departamento de Ciencias de la Actividad Física, Núcleo de Investigación en Salud, Actividad Física y Deporte, Universidad de Los Lagos, Osorno, Chile.

${ }^{4}$ Escuela de Kinesiología, Facultad de Ciencias de la Rehabilitación. Universidad Andres Bello, Viña del Mar, Chile.

${ }^{5}$ Escuela de Educación, Universidad Viña del Mar, Chile.

${ }^{6}$ Escuela de Nutrición y Dietética, Facultad de Medicina, Universidad Andres Bello, Santiago, Chile.

${ }^{7}$ Grupo IRyS, Escuela de Educación Física, Pontificia Universidad Católica de Valparaíso, Chile.
} 
juego pueden llevar a diferencias incluso en este mismo componente. Un estudio llevado a cabo por Holway \& Garavaglia (2009) observó que los forwards tienen mayor masa muscular que sus pares backs. Cabe destacar que los forwards suelen realizar un mayor número de jugadas de contacto con el oponente que los backs (Docherty et al., 1988). En el componente adiposo, los backs han presentado menores valores que los forwards (Gabbett et al., 2010; Darrall-Jones et al., 2016; La Monica et al., 2016). Un aspecto favorable, considerando que éstos deben cubrir mayores distancias que los forwards, las cuales son realizadas a una elevada intensidad (resistencia anaeróbica). Gabbet et al., (2010) observaron que rugbistas élite presentaron un menor grosor de pliegues cutáneos vs la no élite, demostrando que el nivel deportivo es determinante en destacar esta característica física. Recordemos que un elevado componente adiposo, actuará como lastre, lo cual provocará un incremento del gasto energético y una disminución de la aceleración de los movimientos. Estas características son fundamentales para las tareas específicas de alta velocidad que deben cumplir los backs dentro del campo de juego, es decir apoyar al sujeto que traslada el balón, cubrir en defensa y acelerar lejos del scrum, lineout, ruck y maul. En este contexto, un estudio previo demostró que los backs, de distintos niveles competitivos (senior y juveniles), tienen mejor rendimiento que los forwards en piques de 10 m, $20 \mathrm{~m}$ y $40 \mathrm{~m}$ (Gabbett, 2002).

Respecto al somatotipo, utilizando el método de Carter y Heath (Carter, 2002), estudios han demostrado un elevado componente mesomórfico en rugbistas de élite (Carlson et al., 1994; Quarrie et al., 1996; Babic' et al., 2001; Holway \& Garavaglia), lo cual es consistente con el desarrollo de potencia muscular que exigen las acciones durante el juego. Sin embargo, los backs tienen un mayor componente ectomórfico y menor endomórfico que los forwards (Carlson et al.; Quarrie et al.; Babic et al.; Holway \& Garavaglia), lo cual confirma que la adaptación física es específica también a la posición de juego en el rugby.

Ahora bien, es necesario destacar que las diferencias antropométricas observadas en el rugby profesional según la posición de juego, no se mantienen en la mayoría de los rugbistas no profesionales (Babic et al.,; Nicholas, 1997; Ross et al., 2015), demostrando que la especificidad física en este deporte ocurre con niveles altos de competencia. Entonces, si se desea llevar al rugbista hacia un rendimiento deportivo de excelencia, será necesario estudiar y controlar la adaptación morfológica de éste durante todo el periodo deportivo. Asimismo, se confirma que la estrecha relación que existe entre la estructura y función en el deporte competitivo, también se extiende para el rugby.
En los últimos años ha existido un auge del rugby en Chile, desde el año 2015 se han desarrollado nuevas competencias, siendo el torneo nacional de asociaciones de rugby del año 2016 uno de los más importantes para el desarrollo de esta disciplina. Por otro lado, este año es la primera vez que la selección chilena de rugby es invitada a participar del "Campeonato de Rugby de las Américas", donde participan tradicionalmente selecciones de alto nivel tales como Canada, EEUU, Uruguay y Argentina. Sin embargo, el hecho más significativo para el rugby chileno ha sido la clasificación de la selección nacional de rugby 7 al mundial en la ciudad de San Francisco en Estados Unidos, evento que se desarrolló este 2018 donde Chile resultó vencedor del Bowl. En la actualidad, no existe información comprensiva sobre las características morfológicas de rugbistas chilenos. Es por esto, que el objetivo de nuestro estudio fue describir y comparar las características antropométricas morfoestructurales de rugbistas chilenos en relación con la posición de juego.

\section{MATERIAL Y MÉTODO}

Participantes. Una muestra de 121 rugbistas (edad $=24,22 \pm 4,97$ años) pertenecientes a ocho clubes deportivos (Old Navy, Old Mackayans, Villa Alemana Rugby Club, Tralkanes Rugby Club, Halcones Rugby Club, Viña Rugby Club) y 2 universidades (Universidad de Playa Ancha y Universidad Técnica Federico Santa Maria) de la región de Valparaíso en Chile, quienes entregaron su consentimiento para ser medidas sus características físicas generales y variables antropométricas. Estos registros permitieron además obtener la composición corporal y somatotipo con el objetivo de observar las diferencias según la posición de juego. Para los análisis posteriores la muestra fue dividida en forwards $(n=56)$ y backs $(n=65)$. Previo a la firma del consentimiento, los participantes fueron informados detalladamente sobre los procedimientos experimentales y posibles riesgos asociados con su participación en el estudio. Esta investigación fue aprobada por el comité de ética de la Universidad de Playa Ancha, la cual se llevó a cabo siguiendo las recomendaciones de la declaración de Helsinki para estudios con humanos. Al momento de las mediciones, todos los atletas se encontraban sanos, sin antecedentes de enfermedades cardiovasculares o respiratorias. Además, no debían haber consumido café, cigarrillos y/o alcohol durante las 24 horas previas al inicio de las evaluaciones.

Recolección y análisis de datos. Se midió el peso corporal mediante una báscula electrónica (Tanita ${ }^{\circledR}$ HD-357, Japón), la cual presenta una precisión de $0,1 \mathrm{~kg}$. La estatura de pie y sentado se obtuvieron utilizando un papel milimetrado ad- 
herido a la pared y una cinta métrica para ambas, además se utilizó un cajón antropométrico de madera de $40 \mathrm{~cm}$ de alto para la estatura sentado. Los perímetros de cabeza, brazo relajado, brazo contraído, antebrazo, tórax, cintura, cadera, muslo máximo, muslo medio y pierna medial fueron medidos con una cinta antropométrica metálica flexible y no extensible (Lufkin ${ }^{\circledR}$ W606PM, México). Para los pliegues cutáneos tricipital, bicipital, subescapular, cresta iliaca, supraespinal, abdominal, muslo medio y pierna medial se utilizó un calibre de pliegues (SlimGuide, Rosscraft®, USA). Finalmente, para los diámetros biacromial, tórax transverso, tórax anteroposterior, bi-iliocrestidio, humeral y femoral se utilizó un calibre deslizante grande y otro pequeño (Health $\&$ Performance ${ }^{\circledR}$, Chile). Los datos fueron recolectados en una planilla de registro creada en un programa computacional (Excel v. 2007, Microsoft Office ${ }^{\circledR}$, EEUU). Todos los equipos antropométricos fueron previamente calibrados de acuerdo con las recomendaciones de la ISAK (Stewart et al., 2011). La composición corporal se determinó con el método de fraccionamiento en 5 componentes de Kerr, el cuál divide el cuerpo anatómicamente en tejido adiposo, muscular, óseo, piel y residual (Kerr, 1988). Para determinar el Somatotipo se utilizó el método de Carter y Heath (Carter).

Estadística. Para observar la confiabilidad de los parámetros antropométricos determinados por el método Kerr se contrastó el peso corporal estimado y el peso corporal bruto, obteniéndose el coeficiente de correlación intraclase (CCI), coeficiente de correlación de Pearson (r) y coeficiente de determinación $\left(\mathrm{r}^{2}\right)$. Por otro lado, se observó la concordancia a través del método de Bland \& Altman (2003). Entonces, se aceptó la concordancia cuando al menos un $95 \%$ de las diferencias individuales entre el peso corporal estimado y peso corporal medido se encontró dentro del rango de las desviaciones estándar. Todas las variables registradas son presentadas como promedio y desviación estándar. Para observar las diferencias en las características físicas básicas, medidas antropométricas, composición corporal y somatotipo entre los grupos se utilizó la prueba t de Student, ya que las variables presentan varianzas homogéneas. Para homogeneidad se utilizó la prueba F. Para determinar si existieron diferencias entre cada componente del somatotipo por grupo se utilizó la prueba t de muestras independientes, entonces el nivel de significancia estadística elegido fue $p$ $<0,05$. Para comparar la clasificación del somatotipo se utilizó la prueba SANOVA, considerando un valor de significancia estadístico $\mathrm{p}<0,01$. Todos los análisis estadísticos, excepto SANOVA, se realizaron utilizando el programa estadístico GraphPad Prism 6.01 para Windows (GraphPad Software Inc.). Para el análisis SANOVA se utilizó el programa de cálculo del somatotipo (Somatotype Calculation and Analysis Software versión ${ }^{\circledR}$, versión 1.1, USA).

\section{RESULTADOS}

En la Figura 1A se muestra una alta asociación entre peso corporal estimado a través del método antropométrico de Kerr y el peso corporal medido con un CCI= 0,98 (IC 95 $\%=0,96-0,99)$ y $\mathrm{r}=0,98(\mathrm{p}<0,01)$. Además, este método fue capaz de determinar con una precisión del $96 \%$ el peso real del sujeto $\left(r^{2}=0,96\right)$ con $\mathrm{p}<0,01$. El método de BlandAltman (Fig. 1B) determinó que existe concordancia entre el peso estimado y medido donde un $98,3 \%$ de las diferencias se ubicaron dentro de los límites de la desviación estándar. Este método mostró un error constante de $0,53 \mathrm{~kg}$ y una desviación estándar de 2,9kg.

Respecto a las características físicas básicas, los forwards tuvieron promedios mayores de estatura de pie,
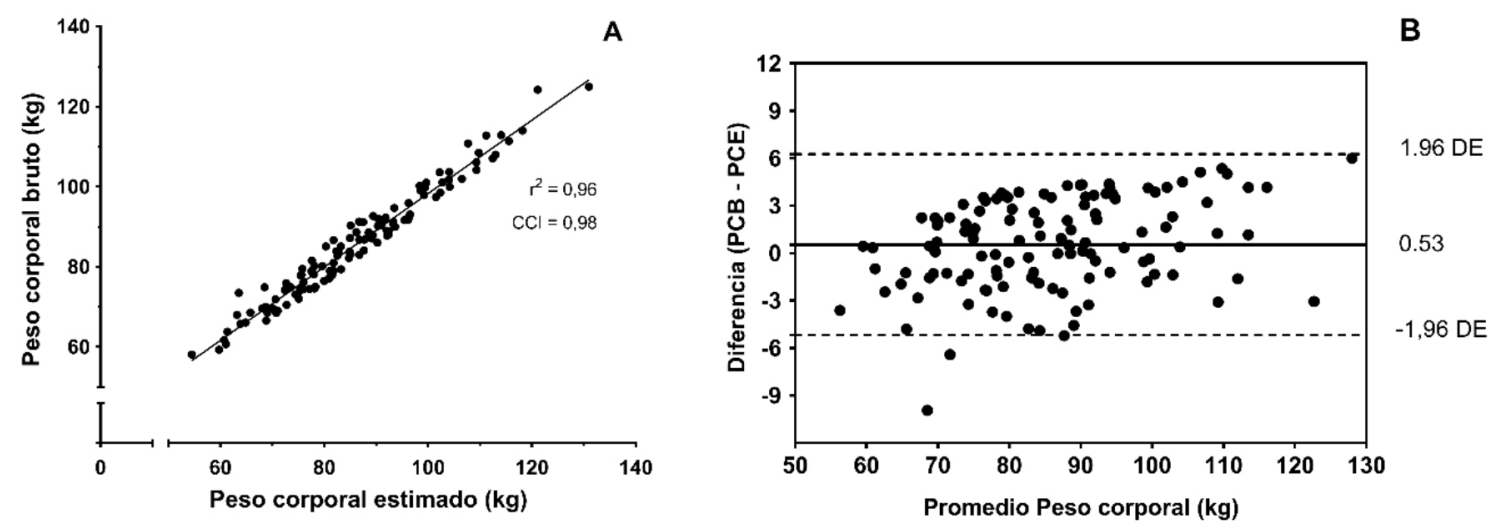

Fig. 1. A) Indicadores de confiabilidad y B) concordancia modelo kinantropométrico pentacompartimental de Kerr (BlandAltman). A) r= coeficiente de correlación de Pearson; $r 2=$ coeficiente de determinación CCI= coeficiente de correlación intraclase. $\mathrm{B}$ ) $\mathrm{PCB}=$ peso corporal bruto; $\mathrm{PCE}=$ peso corporal estimado; $\mathrm{DE}=$ desviación estándar. 
estatura sentado, peso corporal e IMC que los backs con $\mathrm{p}$ $<0,01$ (Tabla I). En esta Tabla I también se observa que, a pesar de no haber diferencias en los promedios individuales de los pliegues cutáneos, perímetros o diámetros entre los grupos, la $\sum$ de 8 pliegues cutáneos fue mayor en forwards que los backs. $(\mathrm{p}<0,01)$.

La Tabla II muestra que existió un mayor tejido muscular y adiposo para los forwards respecto a los backs ( $p$ $<0,01)$. Para el somatotipo, los forwards mostraron los componentes endomorfía y mesomorfía más elevados, y de ectomorfía más bajo, respecto a los backs ( $\mathrm{p}<0,01)$.
En las Figuras 2A y 2B se observa el promedio y dispersión de puntos individuales del somatotipo de los 121 rugbistas según la posición de juego. Además, en las Figuras $2 \mathrm{C}$ y $2 \mathrm{D}$ se observan los porcentajes de distribución según zonas de clasificación del somatotipo para los backs y forwards, respectivamente. Aquí, un $6 \%$ de los backs se clasificaron como mesomorfo-endomorfo, un $72 \%$ endomesomórfico, $11 \%$ mesomórfico balanceado y $5 \%$ como ecto-mesomórfico. Por otro lado, los forwards tuvieron un $16 \%$ de jugadores clasificados como mesomorfoendomorfo, un $71 \%$ endo-mesomórfico, $4 \%$ mesomórfico balanceado y $5 \%$ ecto-mesomórfico.

Tabla I. Promedios y desviaciones estándar de las características generales, físicas básicas y específicas de todos los rugbistas y según posición de juego.

\begin{tabular}{|c|c|c|c|c|c|c|c|c|c|}
\hline \multirow{2}{*}{$\begin{array}{c}\text { Variables } \\
\text { Características fisicas básicas y generales }\end{array}$} & \multicolumn{3}{|c|}{ Forwards $(n=56)$} & \multicolumn{3}{|c|}{ Backs $(n=65)$} & \multicolumn{3}{|c|}{ Total $(n=121)$} \\
\hline & PROM & \pm & $\mathrm{DE}$ & PROM & \pm & $\mathrm{DE}$ & PROM & \pm & $\mathrm{DE}$ \\
\hline Edad (años) & 24,6 & \pm & 5,3 & 23,9 & \pm & 4,7 & 24,2 & \pm & 5 \\
\hline Est atura (cm) & 177,1 & \pm & 6,6 & 171,3 & \pm & $5,6^{*}$ & 173 & \pm & 6,7 \\
\hline Estatura sentado $(\mathrm{cm})$ & 92,9 & \pm & 3,4 & 90,1 & \pm & $3,4^{*}$ & 91,4 & \pm & 3,6 \\
\hline Peso corporal (Kg) & 94,8 & \pm & 12,4 & 77,4 & \pm & $9,1 *$ & 85,5 & \pm & 13,8 \\
\hline $\operatorname{IMC}\left(\mathrm{kg} / \mathrm{m}^{2}\right)$ & 30,3 & \pm & 4,2 & 26,3 & \pm & $2,6 *$ & 28,2 & \pm & 3,9 \\
\hline$\sum$ de 6 pl iegues $(\mathrm{mm})$ & 109 & \pm & 37,5 & 81,7 & \pm & 27,9 & 94,4 & \pm & 35,3 \\
\hline$\sum$ de 8 pl iegues $(\mathrm{mm})$ & 145,9 & \pm & 47,6 & 110,8 & \pm & $36,9 *$ & 127 & \pm & 45,5 \\
\hline \multicolumn{10}{|l|}{ Pliegues cutáneos (mm) } \\
\hline Tríceps & 14,2 & \pm & 5,6 & 10,6 & \pm & 3,6 & 12,3 & \pm & 5 \\
\hline Subescapular & 18,6 & \pm & 9,4 & 13,6 & \pm & 6,2 & 15,9 & \pm & 8,2 \\
\hline Supraespinal & 18,8 & \pm & 8,6 & 13,2 & \pm & 6 & 15,8 & \pm & 7,8 \\
\hline Abdominal & 28,9 & \pm & 10 & 22,9 & \pm & 9 & 25,7 & \pm & 9,9 \\
\hline Mus lo frontal & 16,1 & \pm & 6 & 12,4 & \pm & 4,5 & 14,1 & \pm & 5,5 \\
\hline Pantorrilla & 12,4 & \pm & 5,9 & 9 & \pm & 3,9 & 10,6 & \pm & 5,2 \\
\hline Biacromial & 40,7 & \pm & 1,9 & 39,3 & \pm & 2,2 & 39,9 & \pm & 2,2 \\
\hline Tórax transverso & 30,5 & \pm & 3 & 29,3 & \pm & 2,8 & 29,9 & \pm & 2,9 \\
\hline \multicolumn{10}{|l|}{ Diámetros $(\mathrm{cm})$} \\
\hline Tórax anteroposterior & 23,1 & \pm & 5,1 & 21,5 & \pm & 4,9 & 22,2 & \pm & 5 \\
\hline Biiliocrestidio & 29 & \pm & 2,7 & 26,5 & \pm & 2,4 & 27,7 & \pm & 2,8 \\
\hline Humeral & 7,3 & \pm & 0,5 & 6,9 & \pm & 0,5 & 7,1 & \pm & 0,5 \\
\hline Femoral & 10,3 & \pm & 0,5 & 9,8 & \pm & 0,5 & 10 & \pm & 0,6 \\
\hline Cabeza & 58 & \pm & 1,4 & 56,4 & \pm & 1,4 & 57,1 & \pm & 1,6 \\
\hline Brazo & 35,4 & \pm & 2,8 & 32,3 & \pm & 2,6 & 33,8 & \pm & 3,1 \\
\hline Brazo flexión & 37,7 & \pm & 2,7 & 34,8 & \pm & 2,6 & 36,1 & \pm & 3 \\
\hline Antebrazo & 29,6 & \pm & 1,7 & 27,8 & \pm & 1,5 & 28,6 & \pm & 1,9 \\
\hline \multicolumn{10}{|l|}{ Perímetros $(\mathrm{cm})$} \\
\hline Tórax & 108,6 & \pm & 8,1 & 98,9 & \pm & 6,5 & 103,3 & \pm & 8,7 \\
\hline Cintura & 93,1 & \pm & 10,6 & 83,3 & \pm & 6,9 & 87,8 & \pm & 10,1 \\
\hline Cadera & 106,7 & \pm & 6,6 & 96,8 & \pm & 8,4 & 101,4 & \pm & 9,1 \\
\hline Mus lo medio & 59,4 & \pm & 4,2 & 54,4 & \pm & 3,1 & 56,7 & \pm & 4,4 \\
\hline Pantorrilla & 41,1 & \pm & 2,8 & 37,6 & \pm & 2,8 & 39,2 & \pm & 3,3 \\
\hline Muslo & 65,2 & \pm & 4,6 & 59,7 & \pm & 3,6 & 62,2 & \pm & 4,9 \\
\hline
\end{tabular}

* prueba t de Student significativa respecto a los forwards. 
Tabla II. Promedios y desviaciones estándar de la composición corporal según Kerr y somatotipo de Carter y Heath de todos los rugbistas y según posición de juego.

\begin{tabular}{|c|c|c|c|c|c|c|c|c|c|c|}
\hline \multicolumn{2}{|c|}{ Variable } & \multicolumn{3}{|c|}{ Forwards $(\mathrm{n}=56)$} & \multicolumn{3}{|c|}{ B acks $(\mathrm{n}=65)$} & \multicolumn{3}{|c|}{ Total sujetos $(\mathrm{n}=121)$} \\
\hline \multicolumn{11}{|c|}{ Composicion Corporal Kerr } \\
\hline \multicolumn{11}{|c|}{ Tejido adiposo } \\
\hline & $(\%)$ & 27.5 & \pm & 4.6 & 26.2 & \pm & 5.4 & 26.8 & \pm & 5 \\
\hline & $(\mathrm{Kg})$ & 26.6 & \pm & 6.6 & 20.1 & \pm & $4.9^{*}$ & 23.1 & \pm & 6.6 \\
\hline & Phantom Z & -0.3 & \pm & 1.1 & -1 & \pm & 0.8 & -0.7 & \pm & 1 \\
\hline \multicolumn{11}{|l|}{ Tejido muscular } \\
\hline & $(\%)$ & 46.2 & \pm & 4 & 46.8 & \pm & 4.1 & 46.5 & \pm & 4 \\
\hline & $(\mathrm{Kg})$ & 44.2 & \pm & 6.2 & 36.2 & \pm & $5.5^{*}$ & 39.9 & \pm & 7.1 \\
\hline & Phantom Z & 3.2 & \pm & 1.2 & 2.4 & \pm & 0.9 & 2.8 & \pm & 1.1 \\
\hline \multicolumn{11}{|l|}{ Tejido residual } \\
\hline & $(\%)$ & 11.7 & \pm & 1.6 & 11.9 & \pm & 1.3 & 11.8 & \pm & 1.5 \\
\hline & $(\mathrm{Kg})$ & 11.3 & \pm & 2.5 & 9.2 & \pm & 1.6 & 10.2 & \pm & 2.3 \\
\hline & Phantom Z & 3.4 & \pm & 2.1 & 2.5 & \pm & 1.4 & 2.9 & \pm & 1.8 \\
\hline \multicolumn{11}{|l|}{ Tejido óseo } \\
\hline & $(\%)$ & 9.9 & \pm & 1.2 & 10.3 & \pm & 1.3 & 10.2 & \pm & 1.3 \\
\hline & $(\mathrm{Kg})$ & 9.5 & \pm & 1.3 & 7.9 & \pm & 1.3 & 8.7 & \pm & 1.5 \\
\hline & Phantom Z & -1.3 & \pm & & -1.7 & \pm & 0.7 & -1.5 & \pm & 0.9 \\
\hline \multicolumn{11}{|l|}{ Tejido piel } \\
\hline & $(\%)$ & 4.6 & \pm & 0.4 & 5.1 & \pm & 0.4 & 4.9 & \pm & 0.5 \\
\hline & $(\mathrm{Kg})$ & 4.4 & \pm & 0.3 & 3.9 & \pm & 0.3 & 4.1 & \pm & 0.4 \\
\hline \multicolumn{11}{|c|}{ Somatotipo Carter y Heath } \\
\hline \multicolumn{11}{|c|}{ Componentes } \\
\hline & Endomorfismo & 4.78 & \pm & 1.75 & 3.7 & \pm & $1.38^{*}$ & 4.2 & \pm & 1.65 \\
\hline & Mesomorfismo & 6.94 & \pm & 1.29 & 6.05 & \pm & $1.12^{*}$ & 6.46 & \pm & 1.28 \\
\hline & Ectomorfis mo & 0.67 & \pm & 0.79 & 1.17 & \pm & $0.76^{*}$ & 0.94 & \pm & 0.81 \\
\hline & SAM & 1.98 & \pm & 1.16 & 1.75 & \pm & 0.77 & 1.88 & \pm & 0.97 \\
\hline \multicolumn{11}{|l|}{ Clasificación } \\
\hline & & \multicolumn{3}{|c|}{ Endo-Mesomorfo** } & \multicolumn{3}{|c|}{ Endo-Mesomorfo** } & \multicolumn{3}{|c|}{ Endo-Mesomorfo** } \\
\hline
\end{tabular}

* significancia estadística con la prueba $t$ de Student respecto a los forwards; ** diferencia estadística con la prueba SANOVA respecto a los forwards; SAM: Media actitudinal del somatotipo.

$\mathbf{A}$

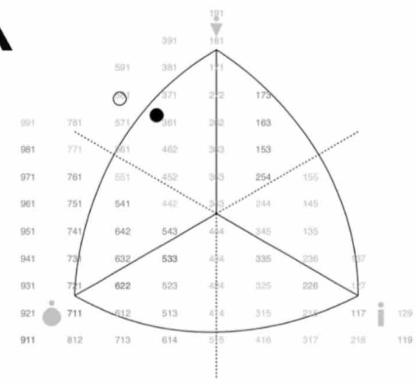

$\mathbf{B}$

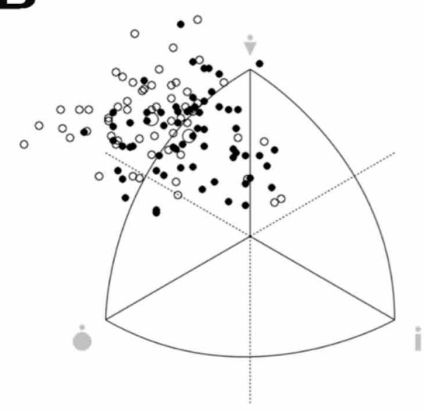

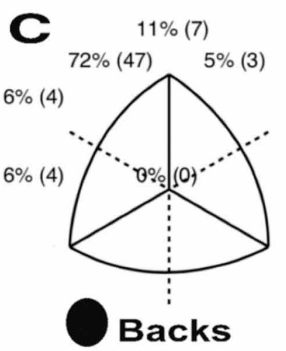

$\mathbf{D}$

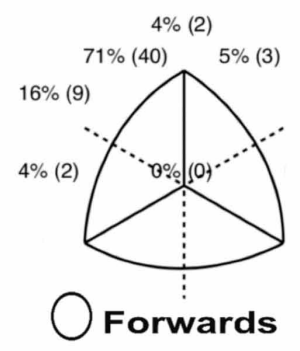

Fig. 2. Somatotipo de rugbistas según la posición de juego. A) somatotipo medio según posición de juego. B) dispersión de los somatopuntos por posición de juego. C) Porcentajes de distribución del somatotipo en backs. D) Porcentajes de distribución del somatotipo en forwards. 


\section{DISCUSIÓN}

Este estudio demostró que existen diferencias significativas en la composición corporal y somatotipo entre los rugbistas chilenos forwards y backs, los cuales fueron obtenidos a través del método kinantropométrico de Kerr (Kerr). Además, este método Kerr resultó ser altamente confiable para los fines utilizados. Un mayor tamaño, peso, y tejidos muscular y adiposo son determinantes físicos en el rendimiento deportivo de los forwards. Según Gabbett (2005), el uso de técnicas estandarizadas, altamente consistentes y precisas como las utilizadas en este estudio, son favorables para identificar patrones morfoestructurales relacionados con éxito deportivo, incluso de acuerdo a sus demandas físicas dependientes de la posición de juego.

Nuestros resultados fueron similares a los encontrados por Lundy et al. (2006) y Cheng et al. (2014) en rugbistas profesionales adultos y de categoría junior respectivamente (Lundy et al.; Cheng et al.) En ambos estudios, una mayor estatura, peso, IMC y sumatoria de 8 pliegues se observó en los forwards respecto a los backs. Lo anterior respalda los resultados obtenidos por Delahunt et al. (2013), quienes demostraron que, en el rugby, los jugadores con mayor masa corporal son propensos a ser utilizados como forwards (Delahunt et al.). Ahora bien, respecto al IMC, este indicador es particularmente alto en los rugbistas, incluso deseable para el rendimiento. El autor también señaló que este indicador puede clasificar erróneamente como obesos a sujetos con bajos porcentajes de tejido adiposo (King et al., 2005). En rugby, la sumatoria de pliegues para observar la acumulación de tejido adiposo es un parámetro muy utilizado. Algunos estudios, han relacionado negativamente un mayor grosor en la sumatoria de pliegues cutáneos con indicadores de rendimiento en el rugby, esto es, intentos de tackle $(r=-0,36)$, tackles completados $(r=0,38)$ y eficacia en los tackles $(r=-0,31)$, destacando la suma de pliegues cutáneos como un indicador determinante en la selección de estos deportistas (Gabbett et al.). En nuestro estudio, sólo la suma de 8 pliegues cutáneos, no así la de 6, mostró diferencias en favor de los forwards respecto a backs. Este grosor de pliegues cutáneos estaría exponiendo un handicap de rendimiento respecto a sus contrincantes deportivos durante la competencia.

Ahora bien, la composición corporal evidenció la presencia de mayor tejido muscular y adiposo absoluto en los forwards, sin observarse cambios en proporción al peso corporal total. Asimismo, el promedio del somatotipo de los forwards en este estudio mostró un mayor componente endomórfico (adiposidad relativa) y mesomórfico (muscularidad relativa) que los backs. Lo anterior, corrobora lo fundamental que es poseer una mayor masa muscular en el rugby, pero con diferentes objetivos según puesto de juego.
Respecto a esto último, la característica de juego de los backs se basa en movimientos rápidos y ágiles para el avance, en cambio en los forwards permitirán contener avances y ganar terreno (La Monica et al.). Por lo mismo, para los backs, más que para los forwards, será necesario tener menores niveles de tejido adiposo debido al recorrido realizado durante la competencia, condiciones que coinciden con las observadas en nuestro estudio. A pesar de esto, los niveles observados de tejido adiposo fueron excesivamente elevados en los forwards (27,5 $\%$ ) y backs (26,2 \%), asociados a un bajo nivel deportivo. Lo anterior se confirma con la distribución en la somatocarta, según los componentes, un mayor porcentaje de rugbistas backs tendieron a acercarse al componente mesomórfico, a diferencia de los forwards cuya tendencia fue hacia el componente endomórfico. Si bien, cada componente promedio del somatotipo difieren entre grupos, la clasificación de todos y por grupo fue la misma, es decir endo-mesomórfico. Esta clasificación es similar a las observadas en estudios previos de rugbistas profesionales adultos (Lundy et al.; Holway \& Garavaglia) y juveniles (Cheng et al.). Sin embargo, los promedios del somatotipo obtenidos en estos estudios muestran mayores componentes de mesomorfía y menores de endomorfía para backs y forwards que los observados en nuestro estudio. La mesomorfía promedio para los forwards en el estudio de Cheng et al. y para los forwards "front row" en Holway \& Garavaglia fue considerada "muy alta" (> 7,5) según Carter y Heath (Carter). A pesar que esto no ocurrió en Lundy et al. el componente mesomórfico observado fue 7,2, muy por encima del 6,94 observado en nuestro estudio, el cual es clasificado como alto (Carter). Es posible que lo anterior tenga relación con las diferencias en el nivel deportivo entre las muestras, ya que nuestro estudio consideró deportistas no profesionales a diferencia de los otros estudios nombrados.

\section{CONCLUSIONES}

Este estudio demostró que la kinantropometría es una herramienta confiable para la estimación de perfiles morfoestructurales en el rugbista. Además la composición corporal de Kerr y somatotipo de Heath \& Carter son herramientas útiles para detectar las principales diferencias morfológicas de los rugbistas según la posición de juego. En este sentido, los rugbistas chilenos tienen un alto componente de tejido muscular y adiposo, sin embargo, en los backs ambos son más bajos que los forwards. Sin embargo, respecto a la clasificación del somatotipo ambos grupos presentaron la misma clasificación endo-mesomórfico. Estas diferencias, confirman la importancia del uso de la kinantropometría para: i) detectar adecuados perfiles morfoestructurales según la demanda del juego, ii) definir posiciones en el campo de juego, y iii) orientar el entrenamiento hacia la consecución de mayores logros deportivos en el rugby. 
BÁEZ-SAN MARTÍN, E.; JIL-BELTRÁN, K.; RAMÍREZCAMPILlO, R.; TUESTA, M.; BARRAZA-GÓMEZ, F.; OPITZ-BEN-HOUR, A. \& YÁÑEZ-SEPÚLVEDA, R. Body composition and somatotype of Chilean rugby players and their relationship with the game position. Int. J. Morphol.,37(1): 331-337, 2019.

SUMMARY: The objective of the study was to describe the morpho-structural characteristics according to playing position, in rugby players from the region of Valparaíso, Chile. Height, weight, body mass index (BMI) and other 25 anthropometric parameters were obtained from 121 competitive rugby players (24.2 \pm 4.9 years), following the recommendations of the International Society for the advancement of Kinanthropometry. The body composition was described in 5 components through the method proposed by Kerr, they are muscle, fat, residual, bone and skin. The somatotype described by Carter and Heath, allowed to describe the components of endomorphy, mesomorphy and ectomorphy. To compare the morphostructural characteristics of the rugby players by playing position, these were separated into forwards and backs. Body weight, sitting and standing height, BMI and S 8-fold were higher in the forwards than the backs ( $\mathrm{p}<0.0001)$. In relation to body mass, adipose tissue and absolute muscle were higher in the forwards than in the backs ( $\mathrm{P}$ $<0.01$ ), but not in the relative. The somatotype showed significant differences in the averages of the three components between forwards 4.8-6.9-0.7 (1.8-1.3-0.8) and backs 3.7-6.1-1, 2 (1.8-1.1-0.8) with P $<0.01$. In conclusion, the playing position in rugby is decisive in the definition of basic physical and morphological characteristics. A higher body weight of the forwards versus backs is influenced by a greater muscle and adipose tissues. Latter was related to a greater mesomorphic and endomorphic component of the somatotype, and lower ectomorphic in the forwards. Despite these differences, both groups are classified as endo-mesomorph.

KEY WORDS: Rugby; Anthropometry; Somatotype; Body composition.

\section{REFERENCIAS BIBLIOGRÁFICAS}

Babic, Z.; Misigoj-Durakovic, M.; Matasic, H. \& Jancic, J. Croatian rugby project-Part I. Anthropometric characteristics, body composition and constitution. J. Sports Med. Phys. Fitness, 41(2):250-5, 2001.

Bland, J. M. \& Altman, D. G. Applying the right statistics: analyses of measurement studies. Ultrasound Obstet. Gynecol., 22(1):85-93, 2003

Brewer, J. \& Davis, J. Applied physiology of rugby league. Sports Med., 20(3):129-35, 1995.

Carlson, B. R.; Carter, J. E.; Patterson, P.; Petti, K.; Orfanos, S. M. \& Noffal, G. J. Physique and motor performance characteristics of US national rugby players. J. Sports Sci., 12(4):403-12, 1994.

Carter, J. E. L. The Heath-Carter Anthropometric Somatotype. Instruction Manual. San Diego, Department of Exercise and Nutritional Sciences, San Diego State University, 2002.

Cheng, H. L.; O'Connor, H.; Kay, S.; Cook, R.; Parker, H. \& Orr, R. Anthropometric characteristics of Australian junior representative rugby league players. J. Sci. Med. Sport, 17(5):546-51, 2014.

Darrall-Jones, J. D.; Jones, B. \& Till, K. Anthropometric, sprint, and highintensity running profiles of English Academy Rugby Union players by position. J. Strength Cond. Res., 30(5):1348-58, 2016.

Delahunt, E.; Byrne, R. B.; Doolin, R. K.; McInerney, R. G.; Ruddock, C. T. $\&$ Green, B. S. Anthropometric profile and body composition of Irish adolescent rugby union players aged 16-18. J. Strength Cond. Res., 27(12):3252-8, 2013.

Docherty, D.; Wenger, H. A. \& Neary, P. Time-Motion Analysis Related To the Physiological Demands of Rugby. J. Hum. Mov. Stud., 14(6):269-77, 1988

Gabbett, T. J. Physiological and anthropometric characteristics of amateur rugby league players. Br. J. Sports Med., 34(4):303-7, 2000.

Gabbett, T. J. Physiological characteristics of junior and senior rugby league players. Br. J. Sports Med., 36(5):334-9, 2002.

Gabbett, T. J. Science of rugby league football: a review. J. Sports Sci., 23(9):961-76, 2005

Gabbett, T. J.; Jenkins, D. G. \& Abernethy, B. Physiological and anthropometric correlates of tackling ability in junior elite and subelite rugby league players. J. Strength Cond. Res., 24(11):2989-95, 2010.

Higham, D. G.; Pyne, D. B.; Anson, J. M. \& Eddy, A. Physiological, anthropometric, and performance characteristics of rugby sevens players. Int. J. Sports Physiol. Perform., 8(1):19-27, 2013.

Holway, F. E. \& Garavaglia, R. Kinanthropometry of Group I rugby players in Buenos Aires, Argentina. J. Sports Sci., 27(11):1211-20, 2009.

Kerr, D. An Anthropometric Method for Fractionation of Skin, Adipose, Bone, Muscle and Residual Masses in Males and Females Age 6 to 77 Years. Tesis de Magister en Kinesiología. British Columbia, Simon Fraser University, 1988.

King, N. A.; Hills, A. P. \& Blundell, J. E. High Body Mass Index is not a barrier to physical activity: Analysis of international rugby players' anthropometric data. Eur. J. Sport Sci., 5(2):73-5, 2005.

La Monica, M. B.; Fukuda, D. H.; Miramonti, A. A.; Beyer, K. S.; Hoffman, M. W.; Boone, C. H.; Tanigawa, S.; Wang, R.; Church, D. D.; Stout, J. R. \& Hoffman, J. R. Physical differences between forwards and backs in American collegiate rugby players. J. Strength Cond. Res., 30(9):238291, 2016.

Lundy, B.; O'Connor, H.; Pelly, F. \& Caterson, I. Anthropometric characteristics and competition dietary intakes of professional rugby league players. Int. J. Sport Nutr. Exerc. Metab., 16(2):199-213, 2006

Nicholas, C. W. Anthropometric and physiological characteristics of rugby union football players. Sports Med., 23(6):375-96,1997.

Norton, K. \& Olds, T. Anthropometrica: A Textbook of Body Measurements for Sports And Health Courses. Sidney, UNSW Press, 1996.

Quarrie, K. L.; Handcock, P.; Toomey, M. J. \& Waller, A. E. The New Zealand rugby injury and performance project. IV. Anthropometric and physical performance comparisons between positional categories of senior A rugby players. Br. J. Sports Med., 30(1):53-6, 1996.

Ross, A.; Gill, N. D. \& Cronin, J. B. Comparison of the anthropometric and physical characteristics of international and provincial rugby sevens players. Int. J. Sports Physiol. Perform., 10(6):780-5, 2015.

Stewart, A.; Marfell-Jones, M.; Olds, T. \& De Ridder, H. International Standards For Anthropometric Assessment. Lower Hutt, International Society for Advancement of Kinanthropometry, 2011.

Dirección para correspondencia:

Eduardo Báez San Martín.

Departamento de Deportes y Recreación

Facultad de Ciencias de la Actividad Física

Universidad de Playa Ancha

Avenida Playa Ancha 850

Edificio Puntángeles

Valparaíso - CHILE

Email: eduardo.baez@upla.cl

Recibido : 07-09-2018

Aceptado: 31-12-2018 